\title{
Japanese Accent Shift: Generalizing from Ambiguous Input
}

\author{
Blake H. Allen and Masaki Noguchi \\ University of British Columbia
}

We address here the question of which phonological generalizations tend to be made when there exist many viable interpretations of a particular set of data. In doing so, this paper contributes to the growing body of research on biases in phonological learning (Wilson 2010; Pater \& Moreton 2012). We pursue this line of inquiry by investigating an under-studied phenomenon in Standard Tokyo Japanese (hereafter Japanese) phonology: vowel hiatustriggered accent shift in verbs (Haraguchi 1996). We argue that due to numerous lexical gaps within the Japanese verbal paradigm, there is no unambiguously superior generalization to be made about which types of verbs exhibit accent shift and which do not. One such generalization we have proposed relies upon sensitivity to a five-point vowel sonority scale. In order to determine which of these possible generalizations native speakers of Japanese have made, we performed a "wug" test (Berko 1958) of novel Yamato (native Japanese) verbs, the methods and results of which are described here.

The results of this experiment reveal that contrary to our expectations, Japanese speakers use phonotactic knowledge about words in the Sino-Japanese lexical stratum in order to make judgments about accent position on our Yamato stratum novel words. Although this finding prevents us from determining whether the sonority-based generalization-which holds true only within Yamato words - has been learned by native speakers, it provides evidence that in the case of phonological ambiguity within one lexical stratum, speakers will refer to extrastratal information to assist in the formation of a phonological generalization. Our results also indicate that words with variable production contribute to the generalization of marked patterns no more than do words with invariably unmarked productions.

\section{Japanese Verbal Accent}

\begin{tabular}{ll|ll}
\multicolumn{2}{c}{ accented verbs } & \multicolumn{2}{c}{ unaccented verbs } \\
bakéru & to be disguised & sitoru & to be soaked \\
habámu & to prevent & susumu & to proceed \\
simésu & to show & tobasu & to fly (trans.)
\end{tabular}

As shown in (1), verbs in Japanese fall into two prosodic classes: those with accent and those without. Accent on verbs in the accented class typically falls on the penultimate mora 
or vowel. A small subset of verbs, however, always exhibit accent on the antepenultimate mora, as in (2). By analogy with the shift of accent leftward away from devoiced high vowels (McCawley 1965; Varden 1998), we refer to this antepenultimate accent pattern as accent shift. As (2) illustrates, all accent shift verbs are of the shape ...VV(C)V; they have no consonant between the antepenultimate and penultimate vowels, placing those vowels into hiatus. However, only ten of these "shift-eligible" verbs always exhibit accent shift.

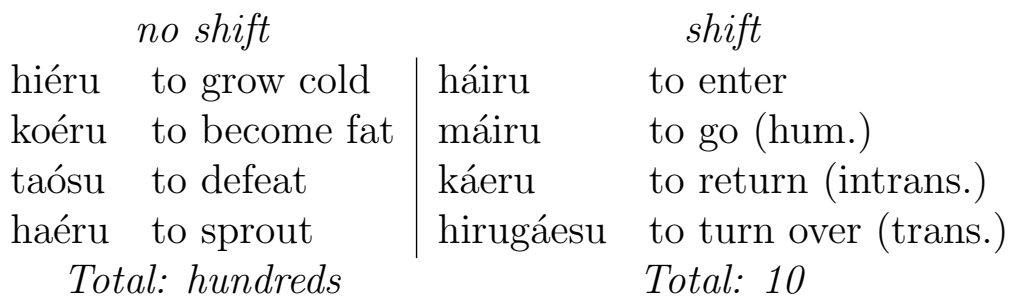

According to Akinaga (2001), approximately two dozen verbs exhibit variable accent shift; both the pronunciation with antepenultimate accent and that with penultimate accent are attested and acceptable.

\begin{tabular}{llll} 
& \multicolumn{3}{c}{ variable shift } \\
osáeru & or & osaéru & to seize \\
otoróeru & or & otoroéru & to weaken \\
kotáeru & or & kotaéru & to answer \\
norikóeru & or & norikoéru & to climb over \\
& \multicolumn{2}{c}{ Total: 24} &
\end{tabular}

Patterns of accent shift based on the antepenultimate-penultimate vowel sequence can be summarized as in (4):

- [ai]: accent is always on $\mathrm{V}_{1}$ (háiru).

- [ae]: for some words accent is always on $\mathrm{V}_{1}$ (káeru); for others, accent is variable (kotáeru/kotaéru); for others, accent is always on $\mathrm{V}_{2}$ (aéru).

- [oe]: for some words accent is variable (otoróeru/otoroéru); for others, accent is always on $\mathrm{V}_{2}$ (koéru).

- others: accent is always on $\mathrm{V}_{2}$.

Consideration of these data raises the following question: what generalization have native speakers of Japanese made about which VV sequences require/allow/disallow accent shift? (5) shows several possible generalizations. The sonority-based generalization, which we have originated in the companion presentation to this article, will be described fully in a forthcoming paper.

1. [ai] will (nearly) always exhibit accent shift; [ae] and [oe] will sometimes shift; all other sequences will show (nearly) no shifting.

2. Same as above, but all VV sequences not observed in real verbs will exhibit accent shift on par with [ae] and [oe]. 
3. A genearlization based on vowel height and/or rounding

4. A sonority-based generalization

Note the one of the key reasons that there exists ambiguity among these possible generalizations is that there are numerous lexical gaps within the Japanese verbal paradigm - nearly half of the twenty-five logically possible vowel combinations are unattested. These gaps present an opportunity to probe knowledge of verbal accent patterns by means of a "wug" test in which judgments about acceptable accent location on these very gaps are tested.

\section{Methods and Results}

We conducted an online questionnaire-based experiment of novel Japanese verbs. The experiment asked subjects to choose between an antepenultimate-accent production and the corresponding penultimate-accent production of each target word using a response scale from 1 (only the first production is acceptable) to 7 (only the second production is acceptable). One novel word was included for each of the twenty-five possible vowel-vowel combinations in Japanese. Several real Japanese words eligible for accent shift were included for verification of subject dialect. We collected responses from thirty-three participants, and two participants' data were removed due to dialectal differences from Standard Tokyo Japanese in the real words.

Responses were coded as ordinal variables, and factor significance $(\operatorname{Pr}<.05)$ was determined by constructing cumulative link mixed models in $\mathrm{R}$ ( $\mathrm{R}$ Core Team 2012). The results were as follows:

1. [ai] strongly tended to exhibit accent shift.

2. [ae], [oe], the non-shift sequences, and the unobserved sequences were all non-significantly different from each other: virtually no shift. (Exceptions in (6.3) below.)

3. [uu], [ee], [ei], [oo], and [ou] were shifted significantly more than any other sequence except [ai].

\section{Discussion}

Results (6.1) and (6.2) demonstrate that the variably-shifting sequences [ae] and [oe] do not cause a generalization of any appreciable amount of accent shift to novel words with those sequences. Broadly, the generalizations made by our subjects are very conservative - they allow accent shift only on sequences like [ai] for which there exist multiple always-shifted words.

Result (6.3) indicates that subjects are using the phonotactics of Sino-Japanese morphemes to inform their judgments about the novel words in our experiment, despite those novel words having the shape of Yamato words. Within a Sino-Japanese morpheme, accentif present - will always be on the first mora. The vowel-vowel sequences in (6.3) comprise exactly the vowel-vowel sequences that exist within Sino-Japanese morphemes (with the 
exception of [ui], which is found in Sino-Japanese morphemes but behaved similarly to nonshifted sequences in our experiment). Hence these judgments reflect the same pattern as [ai]: only and all vowel-vowel sequences across which accent is always on the first mora are considered acceptable loci of accent shift, regardless of the lexical stratum in which those sequences are found. The deviant behavior of [ui] may indicate a preference for accent shift on long vowels rather than complex vowel sequences; note that [ei] and [ou] can be considered allophones of [ee] and [oo].

Our results provide new insight into the role of variable versus categorical lexical patterns in forming phonological generalizations, as well as the interaction between phonotactic properties of different lexical classes. It also speaks to the confounds these issues can introduce in experimental studies. Future studies will need to examine the phenomenon of hiatustriggered accent shift using more and more varied stimuli, and with a greater number of real words for controls, in order to arrive at more detailed conclusions about this phenomenon.

\section{Bibliography}

Akinaga, K. 2001. Shinmeikai Nihongo Akusento Jiten. Sanseido, Tokyo.

Berko, J., 1958. The child's learning of English morphology. Radcliffe College dissertation.

Haraguchi, S. 1996. Syllable, mora and accent. In Phonological structure and language processing, ed. by Takashi Otake \& Anne Cutler, 45-75. New York, NY: Mouton de Gruyter.

MCCAWley, J.D., 1965. The accentual system of standard Japanese. Massachusetts Institute of Technology dissertation.

Pater, J., \& E. Moreton. 2012. Structurally biased phonology: Complexity in learning and typology. Journal of the English and Foreign Languages University, Hyderabad .

R Core Team, 2012. R: A Language and Environment for Statistical Computing. $\mathrm{R}$ Foundation for Statistical Computing, Vienna, Austria. ISBN 3-900051-07-0.

VARDEN, J.K., 1998. On high vowel devoicing in standard modern Japanese: implications for current phonological theory. University of Washington dissertation.

Wilson, C. 2010. Learning phonology with substantive bias: an experimental and computational study of velar palatalization. Cognitive science 30.945-982. 